\title{
Relação da mancha vinho-do-porto com a síndrome de Sturge-Weber: revisão integrativa
}

\author{
Relationship between port wine stain and Sturge-Weber syndrome: integrative review \\ Relación entre la mancha de vino de oporto y el síndrome de Sturge-Weber: revisión \\ integradora
}

Victor Fernando Costa Macedo Noronha ${ }^{1 *}$, Sylvia Kathariny Farias Andrade1, Beatriz Guimarães Ramos $^{1}$, Evlyn Karolayne Bispo Andrade ${ }^{1}$, Thayna Brunelly Dorea Martins ${ }^{1}$, José Aderval Aragão ${ }^{1}$.

\section{RESUMO}

Objetivo: Realizar uma revisão de literatura sobre a correlação entre a mancha vinho-do-porto (MVP) e Síndrome de Sturge-Weber (SSW). Métodos: Trata-se de um estudo de revisão integrativa por meio da seleção de periódicos na base de dados eletrônica PubMed entre 2002 e 2019. Utilizou-se os descritores: "weber sturge syndrome", "stain port wine" e "dermatology". Resultados/Discussão: A SSW é uma síndrome neurocutânea rara, esporádica, congênita e caracterizada por malformações capilares cutâneas, malformações capilares venosas cerebrais e glaucoma. A MVP resulta de mutações somáticas no gene GNAQ durante o desenvolvimento embrionário. Constatou-se que a MVP na área da "testa" possuía maior significância para o desenvolvimento da SSW. Ademais, distribuição mais ampla da MVP, sendo esta unilateral estendendo-se para a maxila e mandíbula e bem como na apresentação bilateral, são considerados fatores de risco. Considerações finais: As características da apresentação dermatológica da MVP determinam se há maior predisposição para SSW. Portanto, faz-se necessário o diagnóstico clínico precoce pela observância da MVP associado aos resultados da ressonância magnética. Tal prática alerta os profissionais sobre os sintomas neurológicos e as complicações dos pacientes portadores da síndrome.

Palavras-chave: Síndrome de Sturge-Weber, Mancha vinho-do-porto, Dermatologia.

\begin{abstract}
Objective: To perform a literature review on the correlation between port-wine stain (PWS) and Sturge-Weber Syndrome (SWS). Methods: This is an integrative review study through the selection of journals in the PubMed electronic database between 2002 and 2019. We used the keywords "weber sturge syndrome", "stain port wine" and "dermatology". Results/Discussion: SWS is a rare, sporadic, congenital neurocutaneous syndrome characterized by cutaneous capillary malformations, cerebral venous capillary malformations and glaucoma. PWS results from somatic mutations in the GNAQ gene during embryonic development. It was found that PWS in the "forehead" area had greater significance for SWS development. In addition, the wider distribution of PWS, being unilateral extending to the maxilla and mandible, as well as bilateral presentation, are considered risk factors. Final considerations: The characteristics of the dermatological presentation of PWS determine whether there is a greater predisposition to SWS. Therefore, early clinical diagnosis is required due to compliance of PWS associated with magnetic resonance imaging results. This practice alerts professionals about the neurological symptoms and complications of patients with the syndrome.
\end{abstract}

Keywords: Sturge-Weber syndrome, Port wine stain, Dermatology.

1 Universidade Tirandentes (UNIT), Aracaju-SE. `E-mail: victornoronha96@gmail.com 


\section{RESUMEN}

Objetivo: Realice una revisión de la literatura sobre la correlación entre la mancha de vino de oporto (MVO) y el Síndrome de Sturge-Weber (SSW). Métodos: Es un estudio de revisión integrador a través de la selección de revistas en la base de datos electrónica PubMed entre 2002 y 2019. Utilizamos las palabras clave "síndrome de esturión de Weber", "mancha de vino de Oporto" y "dermatología". Resultados/Discusión: El SSW es un síndrome neurocutáneo congénito, raro y esporádico caracterizado por malformaciones capilares cutáneas, malformaciones capilares venosas cerebrales y glaucoma. MVO resulta de mutaciones somáticas en el gen GNAQ durante el desarrollo embrionario. Se descubrió que el MVP en el área de "frente" tenía mayor importancia para el desarrollo de SSW. Además, la distribución más amplia de MVO, que se extiende unilateralmente hacia el maxilar y la mandíbula, así como la presentación bilateral, se consideran factores de riesgo. Consideraciones finales: Las características de la presentación dermatológica de MVO determinan si hay una mayor predisposición a SSW. Por eso, se requiere un diagnóstico clínico temprano debido al cumplimiento del MVO asociado con los resultados de la resonancia magnética. Esta práctica alerta a los profesionales sobre los síntomas neurológicos y las complicaciones de los pacientes.

Palabras clave: Síndrome de Sturge-Weber, Mancha de vino de Oporto, Dermatologia.

\section{INTRODUÇÃO}

A Síndrome de Sturge-Weber (SSW) é uma síndrome neurocutânea rara, esporádica, congênita e caracterizada por malformações capilares cutâneas (mancha vinho-do-porto), malformações capilares venosas cerebrais (angiomatose leptomeníngea) e glaucoma (ZALLMANN M, et al., 2016). A mancha vinhodo-porto (MVP) resulta de células endoteliais com diferenciação na pele humana com uma dilatação progressiva de vasculaturas imaturas semelhantes a vênulas. No entanto, essa malformação capilar pode existir sozinha ou associada a muitas outras doenças vasculares congênitas além da SSW, como síndrome de Parkes-Weber, síndrome de Klippel-Trenaunay, síndrome de Proteus e malformações arteriovenosas (NGUYEN V, et al., 2019).

A incidência de SSW é desconhecida. Porém, é estimada em 1 em 20.000 a 50.000 nascidos vivos e afeta igualmente bebês do sexo masculino e feminino (DYMERSKA M, et al., 2017; NGUYEN V, et al., 2019; ZALLMANN M, et al., 2016). Já a prevalência da MVP é estimada em 3 a 5 crianças por cada 1.000 nascidos vivos. Aproximadamente $15-20 \%$ das crianças com MVP facial que envolve o ramo trigeminal oftálmico têm maior risco de possuírem SSW (NGUYEN V, et al., 2019).

A SSW surge do distúrbio do desenvolvimento das três camadas germinativas levando a malformações vasculares na face, coroide e leptomeninges (KOWALSKA-BROCKA J, et al., 2019; NGUYEN V, et al., 2019). A fisiopatologia está relacionada a recentes descobertas de mutações somáticas na proteína de ligação a nucleotídeo de guanina, subunidade G alfa q (GNAQ) (R183Q), fosfatidilinositol 3-quinase (PI3K) e ativação das vias de proteína quinase ativada por mitógeno (MAPK) e vias PI3K em lesões cutâneas na SSW e MVP (NGUYEN V, et al., 2019).

Logo, a ideia anteriormente defendida de que o nevus flammeus reflete a distribuição dos ramos trigêmeos deve ser considerada como um erro histórico de acordo com achados moleculares (HAPPLE R, 2019). Na verdade, a MVP está frequentemente distribuída ao longo das divisões oftálmica (V1), maxilar (V2) e mandibular (V3) do nervo trigêmeo (VASANI RJ, et al., 2012).

Aproximadamente $90 \%$ das MVP's estão localizadas na face, seguidos pelo pescoço, tronco e extremidades em menor frequência. A maioria das manchas (cerca de $90 \%$ ) é unilateral. Certas distribuições de MVP's faciais têm maior potencial para associações neurológicas e ocular na SSW (ZALLMANN M, et al., 2018). Vários estudos sugerem que a presença de MVP na testa ou nas pálpebras superiores, dependendo da sua extensão, têm uma chance de $20 \%$ a $50 \%$ de envolvimento cerebral do SSW. No entanto, o conhecimento atual da relação entre o tamanho da mancha e a gravidade da disfunção neurológica naqueles com envolvimento cerebral da SSW é limitado (KOWALSKA-BROCKA J, et al., 2019). 
A síndrome geralmente se manifesta com a MVP, comprometimento neurológico e glaucoma ocular. Ainda pode ocorrer comprometimento funcional da fala, alimentação ou visão nos pacientes com SSW e esse comprometimento depende da localização, tamanho e estágio da MVP (NGUYEN V, et al., 2019).

Além disso, a perda de autoestima e o estresse psicológico geralmente são problemas clínicos significativos nos indivíduos atingidos devido à estigmatização e desfiguração produzidos, principalmente, pela MVP (NGUYEN V, et al., 2019).

De acordo com a classificação da escala Roach, existem três tipos de SSW: tipo I caracteriza-se por angiomas leptomeníngeos e faciais com ou sem glaucoma pré-existente, tipo II apresenta um angioma facial com glaucoma pré-existente e o tipo III: inclui aparecimento de angiomas leptomeníngeos com ou sem a presença de glaucoma (KOWALSKA-BROCKA J, et al., 2019).

A SSW pode ser diagnosticada por sintomas clínicos típicos, aparência facial da MVP e ressonância magnética cerebral (NGUYEN V, et al., 2019). Muitos autores recomendam a triagem por ressonância magnética (RNM) para bebês assintomáticos com MVP em risco para SSW, mas várias questões permanecem sem resposta sobre quais fenótipos da MVP devem ser rastreados para o diagnóstico da SSW (ZALLMANN M, et al., 2016).

Todavia, a RNM, principalmente quando realizada precocemente, pode falhar na detecção da SSW e a falsa segurança com base em uma RNM negativa pode atrasar o reconhecimento e o tratamento das crises (DUTKIEWICZ AS, et al., 2015).

As opções de tratamento para SSW são limitadas. O objetivo principal é minimizar a atividade convulsiva com medicamentos anticonvulsivantes e quando o tratamento médico é falho, o tratamento cirúrgico é considerado. O glaucoma ocular é tratado para reduzir a pressão do fluido intra-ocular (NGUYEN V, et al., 2019).

Os dermatologistas que cuidam de pacientes com MVP devem perguntar sobre qualidade de vida, fornecer suporte e recursos adequados ao discutir opções de tratamento, além de obter autorização para os procedimentos. Logo, tratamento a laser para a MVP pode melhorar as consequências psicológicas associadas (estresse e depressão) (HAGEN SL, et al., 2017).

Portanto, pela SSW poder gerar complicações decorrente da sua expressão clínica, principalmente o comprometimento neurológico, é primordial compreender dados que possam ajudar a prever, e para isso contamos com a forma e extensão das manifestações extracutâneas. A distribuição corporal da MVP associase com o risco de predisposição para SSW.

Logo, as características da apresentação dermatológica da MVP podem auxiliar em uma investigação crítica dos padrões SSW. Destarte, faz-se necessário o diagnóstico clínico precoce pela observância da MVP associado aos resultados da ressonância magnética. Tal prática alerta os profissionais sobre os sintomas neurológicos e as complicações dos pacientes portadores dessa síndrome.

\section{MÉTODOS}

Tratou-se de um estudo com o objetivo de realizar uma revisão de literatura integrativa sobre correlação entre MVP e SSW. Para a realização desta pesquisa procedeu-se as seguintes etapas: seleção do tema e dos descritores, definição da base de dados para busca, identificação do panorama geral do resultado da busca, registro da avaliação geral dos dados coletados, análise dos dados, interpretação dos resultados e apresentação da revisão.

O levantamento bibliográfico foi realizado por meio dos periódicos da plataforma United States National Library of Medicine/National Institutes of Health (PubMed). A busca na base de dados ocorreu em agosto de 2019 por meio da associação dos descritores "weber sturge syndrome", "stain port wine" e "dermatology". A partir disso, foram encontrados 23 periódicos publicados entre os anos de 2002 e 2019. Inicialmente foram lidos todos os resumos para avaliar o panorama geral e classificar as publicações de acordo com sua relevância para a produção científica conforme o objetivo estabelecido. Após a leitura de todos os periódicos na íntegra, foram selecionados os trabalhos que melhor atendiam aos critérios de busca. 
Os critérios de inclusão foram: trabalhos publicados e disponíveis integralmente em bases de dados científicas ou em versões impressas, artigos com aprovação da comunidade científica e periódicos que aborgem sobre fisiopatologia, quadro clínico, diagnóstico sobre a MVP e/ou correlação da MVP com SSW.

Os critérios de exclusão foram: periódicos que eram relatos de casos e que a temática central era sobre o tratamento para MVP. Dessa forma, 13 publicações constituíram a amostra final (Quadro 1).

Quadro 1 - Estudos selecionados para a pesquisa.

\begin{tabular}{|c|c|c|}
\hline Autores & Título do artigo & Periódico \\
\hline Piram M, et al. (2011) & $\begin{array}{l}\text { Síndrome de Sturge-Weber em pacientes com } \\
\text { coloração facial vinho-do-porto }\end{array}$ & Pediatric Dermatology \\
\hline $\begin{array}{l}\text { Melancon JM, et al. } \\
\text { (2012) }\end{array}$ & $\begin{array}{l}\text { Mancha vinho-do-porto facial: quando se } \\
\text { preocupar? }\end{array}$ & Pediatric Dermatology \\
\hline Vasani RJ, et al. (2012) & $\begin{array}{l}\text { Lesões vasculares cutâneas e seu manejo em } \\
\text { ambiente indiano }\end{array}$ & Dermatologic Therapy \\
\hline Waelchli R, et al. (2014) & $\begin{array}{c}\text { Nova classificação vascular de manchas vinho- } \\
\text { do-porto: melhorando a previsão do risco Sturge- } \\
\text { Weber }\end{array}$ & $\begin{array}{l}\text { British Journal Of } \\
\text { Dermatology }\end{array}$ \\
\hline $\begin{array}{l}\text { Haggstrom AN e } \\
\text { Frieden IJ (2014) }\end{array}$ & $\begin{array}{c}\text { Padrões de marcas de nascença vasculares: } \\
\text { perguntas e pistas }\end{array}$ & $\begin{array}{l}\text { British Journal Of } \\
\text { Dermatology }\end{array}$ \\
\hline $\begin{array}{l}\text { Dutkiewicz AS, et al. } \\
\qquad(2015)\end{array}$ & $\begin{array}{l}\text { Estudo prospectivo de risco para síndrome de } \\
\text { Sturge-Weber em crianças com mancha vinho- } \\
\text { do-porto facial superior }\end{array}$ & $\begin{array}{l}\text { Journal Of The American } \\
\text { Academy Of } \\
\text { Dermatology }\end{array}$ \\
\hline $\begin{array}{l}\text { Zallmann M, et al. } \\
\qquad(2016)\end{array}$ & $\begin{array}{c}\text { Triagem para a síndrome de Sturge-Weber: uma } \\
\text { revisão de ponta }\end{array}$ & Pediatric Dermatology \\
\hline Hagen SL, et al. (2017) & $\begin{array}{l}\text { Qualidade de vida em adultos com manchas } \\
\text { vinho-do-porto faciais }\end{array}$ & $\begin{array}{l}\text { The American Academy } \\
\text { Of Dermatology }\end{array}$ \\
\hline $\begin{array}{l}\text { Dymerska M, et al. } \\
\qquad(2017)\end{array}$ & $\begin{array}{l}\text { Tamanho da marca vinho-do-porto facial de } \\
\text { nascença pode prever resultado neurológico na } \\
\text { síndrome de Sturge-Weber }\end{array}$ & The Journal Of Pediatrics \\
\hline $\begin{array}{l}\text { Zallmann M, et al. } \\
\qquad(2018)\end{array}$ & $\begin{array}{l}\text { Revisão retrospectiva da triagem para síndrome } \\
\text { de Sturge-Weber com ressonância magnética } \\
\text { cerebral e eletroencefalografia em lactentes com } \\
\text { manchas vinho-do-porto de alto risco }\end{array}$ & Pediatric Dermatology \\
\hline Happle R, et al. (2019) & Síndromes com anomalias vasculares da pele & Der Hautarzt \\
\hline Nguyen V, et al. (2019) & $\begin{array}{c}\text { A patogênese da Síndrome de Sturge Weber e } \\
\text { mancha vinho-do-porto: interações complexas } \\
\text { entre alterações genéticas e ativação aberta de } \\
\text { MAPK e PI3K }\end{array}$ & $\begin{array}{l}\text { International Journal Of } \\
\text { Molecular Sciences }\end{array}$ \\
\hline $\begin{array}{l}\text { Singh AK e Keenaghan } \\
\qquad M(2019)\end{array}$ & Síndrome de Sturge-Weber & $\begin{array}{l}\text { National Center for } \\
\text { Biotechnology } \\
\text { Information Search } \\
\text { database }\end{array}$ \\
\hline
\end{tabular}

Fonte: Noronha VFCM, et al., 2019. 


\section{RESULTADOS E DISCUSSÃO}

Segundo Nguyen V, et al. (2019), a MVP é uma malformação multifatorial que envolve a vasculatura e outras estruturas dentro da derme. É considerada uma das anomalias vasculares congênitas mais comuns, que se apresenta ao nascimento e persiste por toda a vida. O fenótipo primário da lesão vascular decorre do espessamento das paredes dos vasos associado à proliferação de pericitos e da duplicação de membranas basais sem ectasia significativa e o fenótipo secundário ocorre por conta da dilatação dos vasos sanguíneos.

Essas vênulas com fenótipo e morfologia anormais co-expressam marcadores que interferem no desenvolvimento e diferenciação das arteríolas e vênulas dérmicas, como os de células-tronco (CD133 e CD166), o venoso (EphB1) e o arterial (EpfnB2). Os dados sugerem que os vasos sanguíneos da MVP são imaturos, com propriedades de estatura aberrante e dupla identidade venosa e arterial.

Evidências recentes mostram que a patogênese da MVP e SSW possui grande participação genética por meio das mutações somáticas nos genes GNAQ (R193Q) e PI3K, que desregulam a sinalização vascular e alteram o desenvolvimento embrionário humano. Logo, se a mutação do mosaico no GNAQ ocorrer no início do desenvolvimento embrionário, pode afetar a vasculatura que está em formação no sistema nervoso central, olhos e pele.

As implicações desses achados são que uma mutação precoce no GNAQ pode afetar um tipo de célula que migra dentro dos placódios faciais afetados e não ao longo de dermátomos. Dymerska M, et al. (2017), em seu estudo, corrobora essa afirmação, tornando desatualizada a teoria dermátomo trigeminal em que a distribuição da MPV ocorreria de acordo com a localização do nervo trigêmeo. Também, Waelchli $R$, et al. (2014) afirma que distribuição da MVP facial parece seguir a vasculatura embriológica da face e que essa nova classificação melhora a previsão de SSW com base no fenótipo da mancha facial.

Nguyen V, et al. (2019) afirma que a MVP aparece inicialmente como máculas vermelhas achatadas na infância. As lesões inicialmente moles tendem a escurecer progressivamente para púrpura com hipertrofia dos tecidos moles e, na meia idade, muitas vezes aumentam como resultado do desenvolvimento de nódulos vasculares suscetíveis a sangramento espontâneo ou hemorragia.

Também, pelas lesões serem lisas e rosas na aparência inicial, podem ser confundidas com uma contusão. Ademais, no mesmo estudo, o autor traz que aproximadamente 15 a 20\% das crianças com SPW facial dermatomal oftálmica (V1) estão em risco de SSW, um distúrbio neurocutâneo com malformações vasculares no córtex cerebral, no mesmo lado da SPW facial.

De acordo com Waelchli R, et al. (2014), as MVP's faciais geralmente são achados isolados. Contudo, quando associadas com malformações vasculares cerebrais e oculares, fazem parte da tríade clássica da SSW. Os primeiros estudos sobre a relação entre o fenótipo da MVP facial e o risco de SSW implicaram na área oftalmológica (V1), que corresponde a uma divisão do nervo trigêmeo com distribuição bilateral e lesões que afetam as pálpebras superiores.

Porém, em estudos mais recentes, ao comparar o fenótipo da MVP com fatores cerebrais e o fenótipo ocular, mostraram que a bilateralidade e distribuição em V1 parecem ser um fator de risco. Ademais, uma MVP mais extensa com envolvimento adicional de V2 e/ou V3 do nervo trigêmeo contribui para a suspeita de SSW. Segundo Piram M, et al. (2011), a SSW associou-se significativamente ao envolvimento palpebral superior, com extensão aos territórios contralateral (40\%) ou proximal homolateral (V1 e V2 ou V1, V2 e V3; $80 \%$ ), e, quando a MVP afeta essas áreas, os médicos devem estar cientes do maior risco de envolvimento neurológico e ocular.

Conforme Nguyen V, et al. (2019), em um estudo de 310 pacientes com MVP, $32 \%$ dos pacientes apresentaram MVP na área inervada pelo ramo maxilar (V2) do nervo trigêmeo, $41 \%$ nos ramos combinados V1 e V2, $5 \%$ nos ramos combinados maxilar e mandibular (V3 ) e 10\% em todas as sucursais. O mesmo estudo também mostrou que, quando o MVP envolveu todos os três ramos, havia uma probabilidade significativamente maior de complicações oculares e/ou do sistema nervoso central, como ocorre na SSW. O estudo realizado por Dutkiewicz AS, et al. (2015) entre 2006 e 2013 com 71 crianças menores de 1 anos de idade em tratamento de MVP facial, mostrou que 51 pacientes não apresentaram nenhum sinal de SSW. 
Logo, o diagnóstico da síndrome ocorreu em 11 crianças (16,6\%) com sinais e crises cerebrais identificadas com auxílio da RNM. Verificou-se que quatro pacientes adicionais apresentavam anormalidades leves em sua primeira ressonância magnética, mas não foi possível determinar a presença definitiva de angioma leptomeníngeo (considerado um grupo suspeito de SSW).

No mesmo estudo verificou-se que três locais específicos (cruzamento da linha média, área temporal e área do nariz) estão associados significativamente a um risco aumentado de desenvolver SSW. Tal dado confirma que o padrão hemifacial clássico está associado a um risco aumentado de SSW.

Também, observou-se uma relação da topografia (extensão da linha média, couro cabeludo, nariz e área temporal) com risco de desenvolver a síndrome. Das crianças com diagnóstico de SSW, 5 tiveram envolvimento de segmento único e 6 com envolvimento de múltiplos segmentos.

Para Haggstrom AN e Frieden IJ (2014), o risco para a SSW também é reconhecido como sendo mais alto nas MVP's com distribuição mais ampla, por exemplo, unilateral estendendo-se para a maxila e mandíbula, bem como na apresentação bilateral. Contudo, para Waelchli R, et al. (2014), a distribuição bilateral não foi um fator de significância independente.

Perceber as características da apresentação dermatológica da MVP pode nos ajudar a prever a predisposição para a SSW. Segundo Waelchli R, et al. (2014), há uma importância na correlação entre acometimento da área V1 do nervo trigêmeo e a SSW.

No entanto, em estudo de Haggstrom AN e Frieden IJ (2014) não foi observada significância nessa distribuição clássica em V1 como fator de risco para SSW, mas, ao invés disso, identificaram a área que se estende do meio da cabeça até o templo, conhecida como "testa", como forte preditor de SSW.

A "testa", que inclui áreas inervadas por todos três ramos nervosos trigêmeos, corresponde ao frontoplacóide nasal, que representa vasculatura e crista neural com células originárias do cérebro anterior e do mesencéfalo em desenvolvimento, fornecendo um elo entre as repercussões cutâneas e neurológicas na SSW.

Dymerska M, et al. (2017) mostra que as MVP's de nascença localizadas na "testa" ou em pálpebras superiores tem $20 \%$ a $50 \%$ de chance de envolvimento cerebral, e que o lado da lesão possui feedback positivo com o lado da disfunção neurológica. A justificativa para esse achado é que uma das áreas citadas, a "testa", envolve todas as três divisões do nervo trigêmeo e corresponde ao desenvolvimento do sistema vascular embrionário do rosto.

É de extrema importância que a MVP verdadeira não seja confundida com a mancha de salmão (naevus simplex). O naevus simplex é uma lesão capilar funcional transitória que ocorre em cerca de $40 \%$ dos recémnascidos e que se apresenta mais comumente como mácula rosa simétrica de borda irregular sobre a linha média do pescoço, "testa" ou pálpebras superiores.

As características oftalmológicas são lesões venosas aumentadas que afetam conjuntiva, episclera, retina e/ou coroides, além de estarem associadas a glaucoma, descolamento de retina e hemorragia coroidal. $O$ glaucoma pode inclusive estar presente desde o nascimento de acordo com Waelchli $R$ et al. (2014) e segundo Nguyen V, et al. (2019) o risco de glaucoma aumenta em até 50\%, quase sempre ipsilateral à SPW facial.

No estudo de Piram, et al. (2011), o glaucoma congênito estava presente em todos os casos com envolvimento do $\mathrm{V} 1$. Todavia, outros acometimentos também se manifestam com maior frequência em pacientes com lesão concomitante de V1 e V2. Para Piram M, et al. (2011), o glaucoma nem sempre é ipsilateral ao MVP facial. Assim, pacientes com uma mancha do território V1, especialmente se extensa, devem ser submetidos a exame oftalmológico bilateral precoce.

Dymerska M, et al. (2017) observou que os indivíduos sem MVP facial e com envolvimento cerebral no SSW têm início mais tarde das convulsões e melhores resultados neurológicos, sugerindo que o envolvimento cerebral é diferente naqueles pacientes do que naqueles com a lesão facial. Consequentemente, pacientes com extenso envolvimento bilateral da MVP e envolvimento cerebral estão em risco particular de resultados neurológicos graves. 
No estudo de Piram M, et al. (2011), o risco de convulsão com MVP facial na ausência de SSW é o mesmo da população em geral e na presença da SSW a frequência das convulsões são mais altas (73,3\%), mas nem todos os pacientes com síndrome desenvolveram quadro convulsivos. Todavia, as convulsões ocorreram em todos os pacientes com SSW com envolvimento de V1, V2 e V3. Ademais, Piram et al. (2011) afirma que quanto mais extenso o PWS, maior o risco de convulsões.

Com o uso da tomografia computadorizada (TC) e RNM puderam ser descritas as características neurológicas revelando angiomatose das leptomeninges, atrofia e calcificação do hemisfério cerebral afetado, ausência de veias corticais e/ou veias de drenagem profunda dilatadas e malformações vasculares envolvendo o plexo coroide. Esses exames complementares e a observação da MVP são cruciais para instigar precocemente e, quando apropriado, iniciar a terapia profilática.

Segundo Nguyen V, et al. (2019), a MVP pode ser diagnosticada prontamente com base na localização anatômica e na aparência característica da lesão. No entanto, por poder associar-se a muitas outras malformações vasculares congênitas, como SSW, síndrome de Parkes-Weber, síndrome de KlippelTrenaunay, síndrome de Proteus e malformações arteriovenosas, a lesão precisa ser examinada por sistemas de imagem, como Doppler, TC ou RNM. Tais métodos podem ser úteis na determinação de possíveis malformações vasculares localizadas em tecidos profundos, como na SSW. No entanto, até $23 \%$ dos pacientes com SSW podem apresentar resultados de ressonância magnética falso-negativos.

De acordo com Zallmann M, et al. (2018), existe o consenso que se deve rastrear crianças com MVP de alto risco. Todavia, a ressonância magnética negativa não pode ser considerada conclusiva se realizada precocemente e não há consenso sobre quem rastrear ou a idade em que uma varredura negativa pode excluir de maneira confiável o SSW. Para Piram M, et al. (2011), a neuroimagem deve ser realizada precocemente se ocorrer convulsão, caso contrário, sugere que o exame deve ser realizado após 1 ano de idade.

Para Dymerska M, et al. (2017) o tamanho da MVP está associada com a gravidade do envolvimento cerebral observado na RMN. Uma exceção ao padrão geral dessa correlação é a lesão de proeminência frontonasal. No estudo, esses pacientes apresentavam MVP relativamente pequena no meio da testa e, no entanto, apresentaram altos escores de anormalidade na RNM e altos escores de disfunção neurológica, indicando comprometimento neurológico significativo.

O atual tratamento de escolha para MVP conforme Nguyen V, et al. (2019) é o laser pulsado de corante (PDL). Tal tratamento consiste na fototermólise seletiva para destruir alvos subterrâneos sem ferir o tecido normal adjacente por dano térmico, ao destruir efetivamente os vasos sanguíneos superficiais da SPW ( $\leq$ $<300 \mu \mathrm{m}$ abaixo da superfície da pele). No entanto, as PDLs não conseguem atingir a temperatura crítica do núcleo necessária para destruir irreversivelmente os vasos sanguíneos sentados em locais mais profundos $(>300 \mu \mathrm{m})$.

\section{CONSIDERAÇÕES FINAIS}

A MVP é decorrente de mutações somáticas que alteram o desenvolvimento vascular durante a formação embrionária. Essa malformação capilar tem forte associação com a SSW e demonstra ser um fator importante na sintomatologia da síndrome de acordo com a sua distribuição. Constatou-se que a MVP tem preferência de acometimento na face. A presença da mancha na fronte e pálpebras é um fator de risco maior para desenvolver a SSW. Portanto, as características da apresentação dermatológica da MVP são de extrema importância para determinar a predisposição para SSW e direcionar o raciocínio clínico dos profissionais em pacientes portadores da síndrome.

\section{REFERÊNCIAS}

1. DUTKIEWICZ AS, et al. A prospective study of risk for Sturge-Weber syndrome in children with upper facial port-wine stain. Journal Of The American Academy Of Dermatology. 2015 mar; 72 (3):473-480. 
2. DYMERSKA M, et al. Size of Facial Port-Wine Birthmark May Predict Neurologic Outcome in Sturge-Weber Syndrome. The Journal Of Pediatrics. 2017 sep; 188:205-209.

3. HAGEN SL, et al. Quality of life in adults with facial port-wine stains. Journal Of The American Academy Of Dermatology. 2017 apr; 76 (4):695-702.

4. HAGGSTROM AN, FRIEDEN IJ. Patterns of vascular birthmarks: questions and clues. British Journal Of Dermatology, [s.I.], v. 171, n. 4, p.693-694, out. 2014.

5. HAPPLE R. Syndrome mit vaskulären Anomalien der Haut. Der Hautarzt. 2019 jul 20; 70 (7):474-480.

6. KOWALSKA-BROCKA J, et al. Sturge-Weber syndrome type II treated with PDL $595 \mathrm{~nm}$ laser. Advances In Dermatology And Allergology. 2015 feb; 32 (1):63-66.

7. NGUYEN V, et al. The Pathogenesis of Port Wine Stain and Sturge Weber Syndrome: Complex Interactions between Genetic Alterations and Aberrant MAPK and PI3K Activation. International Journal Of Molecular Sciences. 2019 may 7. $20(9): 2243$.

8. PIRAM M et al. Sturge-Weber Syndrome in Patients with Facial Port-Wine Stain. Pediatric Dermatology. 2011; 29(1), 32-37.

9. VASANI RJ, et al. Cutaneous vascular lesions and their management in Indian setting. Dermatologic Therapy. 2012; 25(4), 358-375.

10. ZALLMANN M, et al. Screening for Sturge-Weber syndrome: A state-of-the-art review. Pediatric Dermatology. 2016 oct 16; 35 (1):30-42.

11. ZALLMANN M, et al. Retrospective review of screening for Sturge-Weber syndrome with brain magnetic resonance imaging and electroencephalography in infants with high-risk port-wine stains. Pediatric Dermatology. 2018 jul 18; 35 (5):575-581.

12. WAELCHLI R, et al. New vascular classification of port-wine stains: improving prediction of Sturge-Weber risk. British Journal Of Dermatology, [s.I.], v. 171, n. 4, p.861-867, out. 2014. Wiley. 